\title{
Voice hearing over time: A qualitative study of a woman without a psychiatric diagnosis
}

\author{
Anne-Grethe Talseth ${ }^{1}$, Fredricka L. Gilje ${ }^{2 \#}$, Anne Martha Kalhovde ${ }^{3}$ \\ ${ }^{1}$ Department of Health and Care Sciences, Faculty of Health Sciences, University of Tromsø, Tromsø, Norway \\ ${ }^{2}$ College of Nursing, Montana State University-Bozeman, Billings, USA \\ ${ }^{3}$ Department of General Psychiatry, University Hospital of North Norway, Tromsø, Norway \\ Email: anne.g.talseth@uit.no, ${ }^{\#}$ fgilje@bresnan.net, anne.martha.kalhovde@ihelse.net
}

Received 25 September 2012; revised 28 October 2012; accepted 23 November 2012

\begin{abstract}
Voice hearing, a complex perceptual phenomenon, is reportedly common in the general population yet understudied in nursing. Nurses meet voice hearers from diverse cultures; their understandings influence assessment. The aim was to explore voice hearing in everyday life in a non-patient population, revealing insights for assessing voice hearing. Method: Interview data was transcribed, translated into English and analyzed by qualitative content analysis. Outcome measures are thematic descriptions. Results: Voice hearing over time involved past (about year 2000) and recent (2009) time periods. Four main themes were: "Transitioning from not-being-at home when home alone to being at-home", "Shifting overtime from frequent, repetitive voice hearing to inferquent intermittent voice hearing overtime", "Changing from being disempowered to being empowered" and "Progressing from absence of significant others to presence of significant others". Study Limitations: Interviewing a single participant residing in Scandinavia produced in-depth descriptions consistent with the study's aim. The participant had no known psychiatric diagnosis and had not received mental health care when recruited and interviewed. However, we cannot be certain that her voice hearing was or was not non-pathological which raises issues concerning truth value of the study. Conclusions: The results reflect life world existential themes of lived time, lived space, lived body and lived human relation, and offers a framework for guiding and challenging nurses' assessments of voice hearing. Voice hearing can be understood as a bereavement-grief response. Further

\footnotetext{
${ }^{*}$ The authors declare no conflict of interest in the preparation and conduct of this study.

This study received financial support from the Research Council of Norway, University of Tromsø, Tromsø, Norway.

The authors are indebted to the participant who participated in the interview for this study.

${ }^{\#}$ Corresponding author. Retired.
}

research is needed in western and non-western cultures and non-patient populations.

Keywords: Non-Patient; Nursing Research; Western Culture; Life-World Existentials; Voice Hearing

\section{INTRODUCTION}

Voice hearing is a complex perceptual phenomenon [1] viewed differently in western and non-western cultures. In western cultures, it has been related to threatening experiences, such as trauma, distress, deprivation and physical illness [2]. In non-western cultures it has been interpreted as a relatively normal, positive $[3,4]$ or sacred experience [5]. Some mental health personnel feel that inquiring about voice hearing experiences is taboo [6]. With extensive movement of people throughout the world due to, e.g., famine, natural disasters and conflict, nurses encounter diverse cultures in various settings, including persons who experience voice hearing. Of importance is understanding voice hearing, which may or may not involve pathology, and appropriately assessing what is often considered a hallmark of mental illness. Lack of understanding poses risks for inadequate assessment and inappropriate responses. Nurses need to engage with voices hearers, interpreting and discerning meanings of the voices and reliably assessing and offering helpful interventions [7]. The experience of voice hearing is understudied in nursing. Studying voice hearing in a non-patient population may reveal helpful insights.

\section{BACKGROUND}

"Voice hearing", a term used interchangeably with "hearing voices", can be conceptualized as a perceptual phenomenon [8] and understood on a continuum from normality to pathology $[9,10]$. It has often been referred to as pathology (i.e., auditory hallucinations) in nursing [11], medicine, psychiatry [12,13] and psychology [14]. 
Auditory hallucinations, a perceptual phenomenon, are considered a hallmark sign of mental illness [15,16], yet just one-third of those who hear voices have mental illness [17]. While research on voice hearing as auditory hallucinations has often been related to schizophrenia [15, 18], there is little research on how persons with schizophrenia experience their voices [19]. Lack of research interferes with understanding the experience of voice hearing. Using grounded theory, a recent study of a clinical population [1] highlighted the complexity and meaning of voice hearers' experiences. Accessing clinical populations can be difficult; the presence of pathology can complicate communicating such experiences. Studying auditory perceptual phenomenon in non-psychiatric populations may reveal useful insights [16].

"Voice hearing" can also be understood as a normal mental process [20] and a common human experience [21]. Few studies in psychiatry $[10,22]$ and psychology [e.g., 23] focus on hearing voices in non-patient, nonclinical populations. Voice hearing related to bereavement or post-bereavement $[16,24,25]$ has been considered nonpathological.

Blom and Sommer [8] propose re-examining the concept auditory hallucinations, asserting they are not necessarily indicators of pathology. Several movements challenge the pathological view of voice hearing, advocating more humane and holistic understandings. More than 20 networks in the world advocate various ways of understanding voice hearing as a human experience rather than pathology [26]. These include: Hearing Voices Movement established in 1987 in Britain; International Network for Training, Education and Research into Hearing Voices (INTERVOICE) established in 1997 in the Netherlands [27]; and, Hearing Voices Network USA begun in 2010 [26].

Our review of electronic data bases (i.e., CINAHL, Medline, Ovid, Ovid Nursing, SveMed) from 1991 to April 2012 found voice hearing an understudied area of nursing. Search terms included nursing research, voice hearing, hearing voices, non-clinical, auditory hallucinations and epidemiological research. Available published nursing research shows nurses' views or perceptions of and responses to: Voice hearers [28] hearing voices $[7,29]$ inner voice experiences [7], hallucinated voice experiences and atypical sense perception [30]. These studies point to the importance of the quality of the therapeutic relationship to accurate assessment and helpful interventions. Two investigations $[7,28]$ found contrasting views in respondents who were voice hearers with a psychiatric diagnosis or symptoms and nurse respondents, suggesting conflicting realities.

We note lack of published nursing studies about voice hearing or the lived experience of voice hearing in a non-patient population. We concur with England [7,31] and associates [30] that published nursing research about hearing voices in non-patient, non-clinical populations is sparse. In light of various voice hearing movements, a knowledge gap exists about non-patient experiences of voice hearing. Therefore, we embarked on a study to begin to understand the experience of voice hearing in a non-clinical population.

Our aim was to explore "voice hearing" over time in the everyday life of a non-patient. Our intent was to uncover rich, in-depth descriptions of living with "voice hearing". We thought such descriptions may guide and challenge nurses' assessments of voice hearing. We consider that an inner voice within one's thoughts is not voice hearing because one can tell the experience is not an auditory sensation, but rather something in one's thinking [32]. For the purpose of this study, "voice hearing" refers to a subjective auditory sense or sensation of hearing voices unheard by others during wakefulness. The research question was: "What are descriptions of living with 'voice hearing' in a non-patient population?”

\section{MATERIAL AND METHODOLOGY}

The design was qualitative with narrative interviews [33] and qualitative content analysis [34]. The study sample was comprised of one person [35].

\subsection{Selection and Description of Participant}

A purpose sampling was used. Inclusion criteria were: 18 years or older; repeatedly experiencing "voice hearing" for at least one year; and, residing in their own apartment or house. Exclusion criteria were a reported psychiatric diagnosis and receiving psychiatric mental health services and/or treatment.

Recruitment occurred through announcing the project in local newspapers and a flyer posted at a hospital and a university billboard located in Scandinavia during 2009. Four persons responded. One person met inclusion criteria, obtained further information, volunteered to be interviewed and requested the interview occur in her home. The interviewer had no prior contact with the participant.

The participant, a 75-year-old woman, began experiencing voice hearing in 2000. She grew up with her parents and two sisters in a small town in northern Scandinavia and described her childhood as safe and loving. She had two years of post secondary education. Most of her adult life, she resided in northern Scandinavia with her husband and two daughters. She divorced when 50 years old. She had been employed as a cook. She enjoyed social organizations, housekeeping, cooking and knitting which she donated to charity. She reported she had always had mutual, confirming relationships with close family, friends and neighbors. At the time of the interviews, she lived next to her daughter, son-in law and 
two of her three grandchildren. Her medical history included hypertension, past appendectomy and recent knee and hip pain with some immobility. She used a cane for safety. She had no reported psychiatric history.

The first author documented observations of both interviews in a log which comprised approximately two typewritten pages. To assure meeting inclusion criteria, a Mini Mental Status Exam (MMSE), part 1, [36], was conducted by the first author and reviewed by the second author. The score was $16 / 16$, showing orientation to time, place, person and situation, intact recent recall and intact short term memory. Also, she demonstrated a positive self concept and resourcefulness.

\subsection{Data Collection}

The first author conducted two in-depth audio-recorded narrative interviews [33]. Preliminary interpretation of the first interview guided deep exploration in the second interview to develop in-depth descriptions of voice hearing over time. The interviewer holds a $\mathrm{PhD}$ in nursing, has 33 years experience as a psychiatric mental health nurse educator and researcher, has authored various publications and is adept at interviewing. Both interviews were conducted in the participant's home. No other persons were present during the interviews. The first interview occurred January 2009; the second November 2009. The interviews totaled approximately five hours. The interviewer used an interview guide [33] with multiple questions intended to bring forth rich descriptions. The participant was asked to relate voice hearing experiences in her everyday life and describe how she dealt with them. Questions about her thoughts, feelings, actions, time, place and personal relationships related to voice hearing were posed to clarify, explore and understand her descriptions.

The first author transcribed the narrative interviews verbatim. Validation of the interview text involved the first author listening to the audio-recorded text several times while reading and re-reading the text. It was transcribed into English by a professional translator and totaled approximately 60 pages. The first author is bilingual in English and a Scandinavian language; the second author, a US psychiatric nurse educator and researcher, is fluent in English; and, the third author, a psychiatric nurse and Ph.D. student, is fluent in both of these languages.

\subsection{Ethics Considerations}

The project was approved by The Regional Committee for Medical and Health Research Ethics and the Norwegian Social Sciences Data Service (NSD), 2008. The participant gave written consent to participate. She was informed she could withdraw at any time and assured confidentiality in dissemination of results.

\subsection{Data Analysis}

The textual analysis, guided by qualitative content analysis [34] involved an iterative process in which the first two authors participated. Analysis was based on richness and descriptiveness of the text related to the aim, the authors' pre-understandings and life world literature.

The first and second authors read and re-read the whole text to grasp the content and then sorted the textual data. This involved reflecting on the text and dividing it into meaning units, (i.e., words, sentences, phrases related to the aim). Second, these same authors sorted textual data into the following preliminary categories that paralleled the interview questions: Opening up and telling about voice hearing; Telling what happened before hearing voices began; Telling what kind, when and where voices appeared in the past and recently; and telling recent ways to deal with voices. Third, the first two authors formulated the meaning units into condensed meaning units, subthemes and themes. Table 1 reports examples of the condensation-abstraction processes related to past voice hearing. Table 2 reports examples of the condensation-abstraction processes related to recent voice hearing. See Tables 1 and 2.

\section{RESULTS}

The results describe "voice hearing over time" from everyday experience. Textual analysis revealed two points: Past (about the year 2000) and recent (2009). Results of the analysis processes from subthemes to themes related to a past and a recent point in time are reported in Table 3.

The first point in time is past (about the year 2000) voice hearing. The first theme was "Not-being-at-home when home alone". This focused on the participant alone in her bedroom, kitchen or bathroom with unceasing voice hearing. It also included wandering in her house to escape voice hearing while trying to sleep. The voice hearing was never ending as she struggled to escape voice hearing in her personal space. The text revealed:

"You had to get out of bed, you could not remain in bed... I wandered around the house... moved from here to there... to leave and then I moved there to sleep [at her daughters residence], and ... wandered around the house [to escape the voices].

The second theme was "Being disempowered". Descriptions included being dominated by hearing loud, Christian love songs and negative male and female voices identified as relatives. She recognized lyrics but not the voices of loud pleasant songs. The participant felt very annoyed, sad, scared, extremely frightened, worried about losing her mind and concerned about having a psy- 
Table 1. Past voice hearing: Examples of condensation-abstraction processes from meaning unit to condensed meaning unit to subtheme to theme.

\begin{tabular}{|c|c|c|c|}
\hline Meaning unit & Condensed meaning unit & Subtheme & Theme \\
\hline \multicolumn{4}{|l|}{$\begin{array}{l}\text { The voices did not stop } \\
\text { when I was in bed asleep }\end{array}$} \\
\hline $\begin{array}{l}\text { The voices seemed to come } \\
\text { from kitchen ... my bedroom } \\
\text { and ... bathroom }\end{array}$ & $\begin{array}{l}\text { [Alone] in bed with unceasing verbal } \\
\text { voice hearing } \\
\text { Hearing voices in kitchen, bedroom } \\
\text { and bathroom }\end{array}$ & $\begin{array}{l}\text { Never-ending voice hearing in one's } \\
\text { personal space }\end{array}$ & \multirow{3}{*}{$\begin{array}{l}\text { Not-being-at-home when } \\
\text { home alone }\end{array}$} \\
\hline $\begin{array}{l}\text { I was taking a nap in my bed } \\
\text { and heard him singing outside } \\
\text { my window... }\end{array}$ & $\begin{array}{l}\text { [Alone] in bed hearing } \\
\text { singing male voice from outside } \\
\text { Wandered [alone] around house to } \\
\text { escape verbal voice hearing }\end{array}$ & $\begin{array}{l}\text { Struggling to escape voice hearing in } \\
\text { one's personal space }\end{array}$ & \\
\hline $\begin{array}{l}\text { I had to get out of bed and... } \\
\text { wander around the house [to } \\
\text { escape voices] }\end{array}$ & & & \\
\hline
\end{tabular}

Table 2. Recent voice hearing: Examples of condensation-abstraction processes from meaning unit to condensed meaning unit to subtheme to theme.

\begin{tabular}{|c|c|c|c|}
\hline Meaning unit & Condensed meaning unit & Subtheme & Theme \\
\hline $\begin{array}{l}\text { I am not afraid of them [voices]... Now I am used } \\
\text { to them and am very hardened. I am not afraid } \\
\text { anymore... I am not mindful of the fear. } \\
\text { It helped to talk about these things with my family. } \\
\text { I felt more at ease. I said I was fully sane. I was not } \\
\text { making things up... My imagination is not that } \\
\text { wild. That's when they [family] started believing } \\
\text { me. } \\
\text { It was a huge relief. } \\
\text { When I hear voices, I rebuke them, tell them to get } \\
\text { the hell away... } \\
\text {...use my cane, turn TV on just loud enough for me } \\
\text { to hear it,... What I hold in my hand gets thrown at } \\
\text { the wall... To get things [voices] out... I read the } \\
\text { bible. It helps. I also sing songs... Then it [voice] } \\
\text { stops... My anger is short lived. I do all the } \\
\text { chores in the house. I cook dinner and } \\
\text { everything ... a mad person would not be able to do } \\
\text { that... I knit and do different things. }\end{array}$ & $\begin{array}{l}\text { Being convinced, hardened, } \\
\text { unafraid eases fear of voice } \\
\text { hearing } \\
\text { Feeling believed by family } \\
\text { relieves [voice hearing] }\end{array}$ & $\begin{array}{l}\text { Being confident and active } \\
\text { diminishes voice hearing }\end{array}$ & Being empowered \\
\hline
\end{tabular}

Table 3. Themes and subthemes of past voice hearing and recent voice hearing.

\begin{tabular}{|c|c|}
\hline Past voice hearing & Recent voice hearing \\
\hline $\begin{array}{l}\text { Not-being-at-home when home alone } \\
\text { —-Never ending voice hearing in one's personal space } \\
\text { —-Struggling to escape voice hearing in one’s personal room }\end{array}$ & $\begin{array}{l}\text { Being at home when home alone } \\
\text { - Being comfortable in personal space with some voice hearing } \\
\text { strengthens }\end{array}$ \\
\hline $\begin{array}{l}\text { Being disempowered } \\
\text {-Feeling dominated by voice hearing } \\
\text { —-Being certain about lyrics while uncertain about the voice in voice } \\
\text { hearing }\end{array}$ & $\begin{array}{l}\text { Being empowered } \\
\text { —-Being able, confident and acknowledged relieves voice hearing } \\
\text { —Being confident and active diminishes voice hearing }\end{array}$ \\
\hline $\begin{array}{l}\text { Frequent repetitive voice hearing over time } \\
\text { - Hearing incessant music and noise during nighttime and intermittent } \\
\text { voices during daytime }\end{array}$ & $\begin{array}{l}\text { Infrequent intermittent voice hearing over time } \\
\text { - Voice hearing occasionally occurs in autumn, winter and when un- } \\
\text { able to sleep }\end{array}$ \\
\hline $\begin{array}{l}\text { Absence of significant others } \\
\text { —-Distressed relationships sensed within voice hearing } \\
\text { — Not doubting voice hearing (in the past) and not being believed }\end{array}$ & $\begin{array}{l}\text { Presence of significant others } \\
\text { —-Sensing presence of others unburdens } \\
\text { —Disclosure with others who believe unburdens voice hearing } \\
\text { —-Spiritual presence connects with others, relieving voice hearing }\end{array}$ \\
\hline
\end{tabular}


psychiatric illness. She was convinced she repeatedly heard voices. The participant indicated:

"I was scared a lot and frightened. Worried I was losing my mind and getting a psychiatric illness... I was sad, very sad and annoyed".

The third theme was "Frequent repetitive voice hearing over time". Data showed frequent repeated daytime and nighttime voice hearing, with mostly nighttime verbal and musical voice hearing. During daytime, the voice hearing disappeared when she talked to the voices. She heard incessant music and noise during nighttime and intermittent voice during the daytime. Examples are:

"Repeated knocking on my outside wall in the middle of the night..., night music came from the basement".

"The voices seemed to come from the kitchen. I came into the kitchen [in the daytime] to talk to them [the voices] — and suddenly they were gone".

Theme four was "Absence of significant others". This theme reflected negative and positive relationships with family members, such as being very convinced about voice hearing despite disbelief from family and loss of relationship through death of an important family member. An example from the text is:

"A cousin grew up with me ... we lived together... Nobody knew he had brain cancer. He was definitely ill and died... It affected me greatly... He was a very important person [to me]. When he became ill, then I started to hear voices".

The second point in time was recent (the year 2009). Here, the verb tense revealed the "now" of the voice hearing experience in contrast with "then" (i.e., the past). The first theme was "Being at-home when home alone". This focused on the participant alone in her house and strengthened by expressing anger [toward voices] which distanced voice hearing and diminished fear. Distancing voice hearing with anger strengthened her as she became comfortable in her personal space. The participant revealed:

"Whether or not they [voices] are afraid of me now... I become angry which gives me strength... I do not know, but at least they [voices] are gone a lot of the time".

The second theme was "Being empowered". Being accustomed to the voices strengthened her, easing her fear of voice hearing and increasing her confidence. This happened through engaging in distraction (e.g. household chores), actively rebuking the voices and expressing anger, all which diminished fear of voice hearing and increased being believed by her family. An example is:

"It helped to talk about these things with my family. I felt more at ease. In the beginning, they did not believe me... I said I was fully sane. I was not making things up... My imagination is not that wild. That's when they started believing me. It was a huge relief'.

The third theme was "Infrequent intermittent voice hearing over time”. Descriptions included decreased frequency of daytime voice hearing, especially in autumn and winter time, yet night time voice hearing occurred when alone trying to sleep. The participant alleged the following:

"I still hear voices, but not often these days... I hear them a lot less, especially during autumn and winter. Now, it is a long time since I heard those voices... I am still afraid of being alone in the house [at night]... I hear the voices when I am alone [at night]".

The fourth theme was "Presence of significant others". Sensing the presence of meaningful others unburdened her and a spiritual presence connected her with others, relieving voice hearing. This happened through openly telling and being with others who believed voice hearing, which decreased her voice hearing and relieved fear. The participant conveyed:

"It helps to talk about these things [voice hearing]... I feel more at ease. At first my family did not believe me ... then they started believing me... It was a huge relief'.

The 8 themes were synthesized into 4 main themes. These are "Transitioning from not-being-at-home to being-at-home when home alone", "Shifting over time from frequent repetitive voice hearing to infrequent intermittent voice hearing", "Changing from being disempowered to being empowered" and "Progressing from absence of significant others to presence of significant others”. Synthesis of themes to main themes is reported in Table 4. See Table 4.

\section{DISCUSSION}

While the results represent a single participant situated in a locale dominated by a western view of voice hearing as pathological, they pose some insights into the complexity of the voice hearing experience. These results may apply to clinical or non-clinical situations and provide an understanding of a way to assess voice hearing regardless of cultural context.

The results can be understood in light of van Manen's life world existentials [37]. Each main theme reflects one of van Manen's 4 life world existentials; i.e. lived space (spatiality) refers to felt space; lived body (corporality) refers to being bodily in the world; lived time (temporality) refers to subjective time in contrast to objective time; and lived human relation (lived other, relationality or communality) refers to lived relation with other human beings in shared interpersonal space. While these can be studied individually and can guide research processes, they are inseparable, overlapping and interwoven [37]. Collectively they describe an understanding of the structure of our life world.

The first main theme, "Transitioning from not-beingat-home to being at-home", describes voice hearing in terms of spatiality. The lived experience of space in- 
Table 4. Synthesis of themes into main themes.

\begin{tabular}{ll}
\hline Themes & Main themes \\
\hline $\begin{array}{l}\text { - Not-being-at-home when home alone } \\
\text {-Being at-home when home alone }\end{array}$ & $\begin{array}{l}\text { Transitioning from not-being at home when home alone to being at home when home } \\
\text { alone }\end{array}$ \\
-Being disempowered & Changing from being disempowered to being empowered \\
-Being empowered & Shifting overtime from frequent repetitive voice hearing to infrequent intermittent voice \\
- Frequent repetitive voice hearing over time & hearing \\
- Infrequent intermittent voice hearing over time & Progressing from absence of significant others to presence of significant others \\
- Absence of significant others &
\end{tabular}

volved two aspects: Physical space of the participant's bedroom, kitchen or bathroom and emotional space of the participant being alone and feeling loneliness, a sense of being lost. Of note is that, in the past, physical space was often restricted to private space (i.e., bedroom and bathroom) where she was alone and felt lonely. In the recent time period, physical space seemed not only to be limited to certain rooms of her house (bedroom, kitchen, bathroom), but to her house overall. Lived space is "largely pre-verbal, yet it affects the way we feel" [37, p. 102]. Furthermore, our home concerns the "fundamental sense of our being" [37, p. 102]. According to Zingmark and associates [38], the sense of being related was found to be a common condition of the experience of beingat-home (i.e., related to significant others, significant things, significant places, significant activities, one-self and transcendence).

The second main theme, "Changing from being disempowered to being empowered", describes voice hearing in terms of corporality. The lived experience of body involved sensing (e.g., hearing, feeling, believing). In our study, we note voice hearing was mainly, but not solely, situated in the participant's bedroom when trying to sleep. Also, the death of her cousin impacted her tremendously, suggesting voice hearing related to post-bereavement [24, 25]. Religious texts and rituals helped allay her fears, suggesting that a spiritual connection was meaningful to managing voice hearing. Similarly, in a study of voice hearer's who had recovered, Romme and associates [39] found that voice hearers mainly described problems of threats and feelings of powerlessness and anxiety about becoming mad. These authors also found that recovery from distressing voices involved focusing on spirituality and realizing dreams.

Various bodily experiences described in literature generally address voice hearing as non maleficent, or positive, and maleficent, or negative. A recent study [40] found that patient voice hearers experienced both positive and negative content and that voice content was the only significant predictor of emotional distress and the strongest predictor of contact with mental health services. A study of non-patient voice hearers showed they perceived their voices as predominantly positive [22,24,25].
While voice hearing in the nonclinical population is usually positive and nonthreatening, in the psychiatric population it tends to be frequent, intrusive and distressing [10]. An important distinction between voice hearing and hallucinations in schizophrenia is that hallucinatory events are rarely experienced as unpleasant, emotionally threatening or hostile [41]. However, traumatic life events, physical and emotional abuse and auditory hallucinations seem to be linked [11]. In our study, we note bodily experiences associated with voice hearing that are both positive (e.g., being relieved) and negative (e.g., fear and distress). Some literature proposes that many voice hearers show low self esteem affecting their ability to clearly explain their voice hearing experiences [31,42]. Martin [43] found that service users who experienced auditory hallucinations described increased confidence and self esteem from participating in a group which helped them understand their voices. Results of a study [28] showed voice hearers needed to take responsibility for their own experiences; establishing personal control over voices gave hope for future recovery [28]. Selfesteem, personal control and hope are associated with empowerment.

The third main theme, "Shifting over time from frequent repetitive voice hearing to infrequent intermittent voice hearing", describes voice hearing in terms of temporality. In our study, the lived experience of time shifted from repetitive distressing daytime and night time (i.e., clock time) voice hearing to infrequent clock and calendar time voice hearing. However, findings show voice hearing related to naptime, evening and night time sleeplessness. Distress often implies intense, frequent, intrusive worry or anguish. While not reported in this study, research shows voice hearing associated with various neurobiological correlates [e.g. 8], substance abuse [e.g. 44], post critical care [e.g. 45] and neurodegenerative disorders, such as Parkinson's [e.g. 46].

Progressing from "Absence of significant others" to "Presence of significant others", the fourth main theme, describes voice hearing in terms of relationality. This study reveals that the experience of human relations related to voice hearing deals with the presence or absence of relationships. Parse [47] writes that only in "true 
presence” [47, p. 74] can people choose to alter their health pattern. In our study, over time, the participant's absence of relationships with significant others was altered and the presence of significant others emerged as significant others believed her voice hearing. The participant disclosed this as she became confident in trusting the interviewer. Being believed may be understood as experiencing true presence. The interviewer's true presence with the participant brought participant and interviewer into a shared reality. In a service users' group with auditory hallucinations, Martin [43] found reoccurring themes of "not being alone" and "closeness of the group". This means relational outcomes were viewed as beneficial to voice hearers. As described by Zingmark and associates [38], the presence of others who believe voice hearing as well as consoling texts foster "athomeness with self". In our study, it is apparent that, as the participant's relationship with significant others changed, her relationship with her voices also changed. This seemed to reclaim her life. This was also reported by Romme and associates [39] in their study of fifty people who recovered from distressing voice hearing. Relationship is central to empowerment and re-empowerment.

The results of our study are reflected in Coleman's [48] descriptions of four important elements related to recovery from distressful voice hearing. These were: Being involved with others for direction, hope, support and friendship; improving self-esteem, self-confidence, selfawareness and self-acceptance; making responsible choices for personal decisions, finding purpose in life and taking ownership of one's voice-hearing experience.

\subsection{Relevance to Nursing}

Results of this study reveal a contemporary understanding of verbal and musical voice hearing that can guide and challenge nursing assessments. Nurses' assessments affect diagnostic decisions. Commonly held responses to voice hearing included in psychiatric nursing textbooks in the western world [11] are: "Avoiding discussing voices with voice hearers because doing so supports psychopathology" [7, p. 130]; "stopping or blocking voice hearing” [49, p. 97]; and "reinforcing reality which discourages engaging with voice hearing experiences" [27, p. 435]. In contrast, contemporary nursing literature and nursing research advocate a contextual, relational perspective of voice hearing consistent with Romme's and Escher's focus on the "culturally situated social environment" [17, p. 436].

Adam and associates [50] found the interpersonal nature of the relationship with the nurse most valued by voice hearers in contrast to technical aspects of care. England [7] declares that "graduate nursing curricula should require holistic voice hearing assessment and nurses should assess more holistic measures of voice hearing so they can discern uniqueness in voice hearers' experiences" [7, p. 138]. Milham and Easton [49] suggest a nonjudgmental approach, proposing a view of voice hearing as "an experience to accept and make sense of" [49, p. 98]. Martin [43] proposes focusing on uniqueness of each voice hearer and listening to, rather than blocking, subjective experiences.

Of importance is that mental health professionals explore voice hearing experiences with a sense of belief. Essential to nursing assessment is a nonjudgmental attitude, listening to and acknowledging personal experiences and exploring meanings. These approaches require cultural sensitivity. van Manen's [37] life-world existential address fundamental structures of lived-experience, considering social, historical and cultural contexts.

Of significance is that nurses often encounter persons experiencing grief reactions. In our study, the onset of the participant's voice hearing followed the death of a person who meant a lot to her. Nursing research on voice hearing associated with grief is very sparse. One study [51] of bereaved spouses found about half of participants had experienced post bereavement hallucinations, mostly auditory and visual, and sensed the presence of the deceased. A few non-nursing related studies explored grief hallucinations [52,53] and complicated grief [54]. These few studies begin to legitimize an understanding that grief responses can burden persons and manifest in hearing voices. Hearing voices may be a normal response to trauma, unrelated to psychosis and present for many years without indicating a need for mental health care [55].

\subsection{Methodological Considerations}

Guba's [56] model of trustworthiness outlines four criteria for judging trustworthiness.

Truth value, the first criterion, focuses on the subject and credibility of results. Data showed the participants voice hearing was precipitated by a grief reaction and endured 10 years. The participant reported she had seen her general physician and had not sought nor received mental health services or been diagnosed with a psychiatric condition. We acknowledge that it is unknown whether or not her voice hearing was non-pathological, possibly raising issues about the truth value of this study. While neuorbiological correlates have been studied in persons with schizophrenia, such research in non-psychotic persons is quite recent and has been conducted with small samples of highly selected participants with inconsistent results. Diederen and associates [57] reported decreased dopamine dysfunction usually found in schizophrenia is unlikely related to auditory verbal hal- 
lucinations in healthy voice hearers. Neither altered dopaminergic function nor psychotic symptoms were observed or reported in the participant in our study prior to or during the research period. Overtime, she showed no alterations in social, affective or psychological functions that affected her activities of daily living or merited treatment. The participant seemed to present with nonpsychotic, non-evoked auditory verbal hallucinations.

In our study, a form of peer review occurred as the first two authors collaborated in analyzing and interpreting data based on a real-life situation. The third author's critique of analyses and interpretation confirmed the results and provided refinement and clarification. The researchers' backgrounds as psychiatric nurses and nurse educators were explicated, exposing their biases and experiences with the topic. The first author and participant had no contact prior to the interview. Three forms of data were gathered over 11 months; interviews, Mini Mental Status Exam (MMSE) and a log. Member-check was not included.

The second criteria, applicability, relates to applying results to other settings. We see these findings can generate interest in Van Manen's life world existential [37], thus, being useful to other researchers. While this is a sample with one participant it produced in-depth descriptions consistent with the study's aim. These results uncovered a holistic perspective, consistent with a nursing perspective which could be applicable to other settings.

The third criterion is consistency. The researchers provide two tables showing examples of the analyses processes and an audit trail (Refer to Tables 1 and 2). Neutrality is the last criteria. Throughout the study, we reflected on our analyses and interpretive approaches; we openly critiqued each other's writings throughout manuscript development. We claim no conflicts of interest in the conduct of the study.

\section{CONCLUSIONS}

In this study the results show that voice hearing, a lived human experience, can occur in a non-patient population and makes sense when viewed in light of personal history [26], such as loss and grief. The onset of the participant's verbal and musical voice seemed to be bereavement. Her voice hearing diminished as she experienced acknowledgement and understanding, instead of disbelief, from significant others. Understanding and exploring meanings of voice hearing experiences is important. Van Manen's life world perspective provided a helpful framework for articulating an understanding of voice hearing experiences. This may guide nurses' understandings and help, as well as challenge, nurses' assessments of voice hearing.

The experience of voice hearing is a subjective, complex, perplexing and understudied perceptual phenome- non in nursing. Studying auditory perceptual phenomenon in non-patient populations may reveal useful insights [16]. Qualitative methods provide a holistic approach to inquiry appropriate for various populations. Research is needed with cross cultural perspectives, larger sample sizes, mixed genders and ages and approaches that shed light on voice hearing associated with, for example, bereavement-grief. We hope this study inspires other researchers to further describe this phenomenon that is not explored much in nursing.

\section{REFERENCES}

[1] Fenekou, V. and Georgaca, E. (2010) Exploring the experience of hearing voices: A qualitative study. Psychosis, 2, 134-143. doi:10.1080/17522430903191783

[2] Lakeman, R. (2001) Making sense of the voices. International Journal of Nursing Studies, 38, 523-531. doi:10.1016/S0020-7489(00)00101-2

[3] Al-Issa, I. (1977) Social and cultural aspects of hallucinations. Psychological Bulletin, 3, 570-587. doi:10.1037/0033-2909.84.3.570

[4] De Jong, J.T.V.M. (1987) Decent into African psychiatry. Royal Tropical Institute, Amsterdam.

[5] Lukoff, D. (2007) Visionary spiritual experiences. Southern Medical Journal, 6, 635-641. doi:10.1097/SMJ.0b013e318060072f

[6] Deegan, P. (2011) Hearing voices that are distressing: Selfhelp resources and strategies. http://www.power2u.org/articles/selfhelp/voices.html

[7] England, M. (2007) Accuracy of nurses' perceptions of voice hearing and psychiatric symptoms. Journal of Advanced Nursing, 58, 130-139. doi:10.1111/j.1365-2648.2006.04162.x

[8] Blom, J.D. and Sommer, I.E. (2010) Auditory hallucinations: Nomenclature and classification. Journal of Cognitive and Behavioral Neurology, 23, 55-62. doi:10.1097/WNN.0b013e3181b2791e

[9] Serper, M., Dill, C.A., Chang, N., Kot, T. and Elliot, J. (2000) Factorial structure of the hallucinatory experience: Continuity of experience in psychotic and normal individuals. Journal of Nervous and Mental Disease, 193, 265-272. doi:10.1097/01.nmd.0000158374.54513.a0

[10] Beavan, V., Read, J. and Cartwright, C. (2000) The prevalence of voice-hearers in the general population: A literature review. Journal of Mental Health, 20, 281-292. doi:10.3109/09638237.2011.562262

[11] Coffey, M. and Higgon, J. (2001) Auditory hallucinations. Journal of Psychiatric and Mental Health Nursing, 21, 18-22.

[12] Hoffman, R.E. and Varanko, M. (2006) "Seeing voices": fused visual/auditory verbal hallucinations reported by three persons with schizophrenia-spectrum disorder. Acta Psychiatrica Scandinavia, 14, 290-293. doi:10.1111/j.1600-0447.2006.00791.x

[13] Neckelmann, G., Specht, K., Lund, A., Ersland, L., Smie- 
voll, A.I., Neckelmann, D. and Hugdahl, K. (2006) Morphometry analysis of grey matter volume reduction in schizophrenia: Association with hallucinations. International Journal of Neuroscience, 116, 9-23. doi:10.1080/00207450690962244

[14] Davies, M.F., Griffin, M. and Vice, S. (2001) Affective reactions to auditory hallucinations in psychotic, evangelical and control group. British Journal Clinical Psychology, 40, 361-370. doi:10.1348/014466501163850

[15] Hardy, A., Fowler, D., Freeman, D., Smith, B., Steel, C., Evans, J., Garety, P.A., Kuipers, E., Bebbington, P.E. and Dunn G. (2005) Trauma and hallucinatory experience in psychosis. Journal of Nervous and Mental Disease, 193, 501-507. doi:10.1097/01.nmd.0000172480.56308.21

[16] Boksa, P. (2009) On the neurobiology of hallucinations. Journal of Psychiatry Neuoscience, 34, 260-262.

[17] Romme, M. and Escher, S. (1994) Hearing voices. British Medical Journal, 309, 670. doi:10.1136/bmj.309.6955.670a

[18] Romme, M. and Escher, S. (2000) Making sense of voices: A guide for professionals who work with voice hearers. MIND Publications, London.

[19] Gray, B. (2008) Hidden demons: A personal account of hearing voices and the alternative of the hearing voices movement. Schizophrenia Bulletin, 34, 1006-1007. doi:10.1093/schbul/sbn099

[20] Slade, P.D. and Bental, P.R. (1988) Sensory deception: A scientific of hallucination. Johns Hopkins University Press, Baltimore.

[21] Watkins, J. (1998) Hearing voices: A common human experience. Hill of Content Publishers, Melbourn.

[22] Honing, A., Romme, M., Bernardine, E., Sandea, E., Monique, P. and Marten D. (1998) Auditory hallucinations: A comparison between patients and non-patients. Journal of Nervous and Mental Disease, 186, 646-651. doi:10.1097/00005053-199810000-00009

[23] Morrison, A.P., Wells, A. and Nothard, S. (2002) Cognitive and emotional predictors of predisposition to hallucinations in non-patients. British Journal of Clinical Psychology, 41, 259-270. doi:10.1348/014466502760379127

[24] Rees, W.D. (1971) The hallucinations of widowhood. British Medical Journal, 4, 37-41.

[25] Olson, P.R., Suddeth, J.A., Peterson, P.J. and Egelhoff, C. (1985) Hallucinations of Widowhood. Journal of American Geriatric Society, 33, 543-547.

[26] Hornstein, G. and Dillon, J. (2012) Hearing voices network USA. http://www.hearingvoicesusa.org

[27] Romme, M. (2012) International network for training, education and research into hearing voices. http://intervoiceonline.org

[28] Coffey, M. and Hewitt, J. (2008) 'You don' talk about the voices": Voice hearers and community mental health nurses talk about responding to voice hearing experiences. Journal of Clinical Nursing, 17, 1591-1600. doi:10.1111/j.1365-2702.2007.02185.x

[29] Coffey, M., Higgon, J. and Kinnear, J. (2004) Therapy as well as the tablets. An exploratory study of service users "views of community mental health nurses" (CMHNs) responses to hearing voices. Journal Psychiatric and Mental Health Nursing, 11, 435-444. doi:10.1111/j.1365-2850.2004.00740.x

[30] England, M., Rubenstein, T. and Tripp-Reimer, L. (2003) Relevance of cues for assessing hallucinated voice experiences. International Journal of Nursing Terminology Classifications, 14, 77-95. doi:10.1111/j.1744-618X.2003.tb00067.x

[31] England, M. (2005) Mediation of the relationship of inner voice experiences to health-related quality of life. Perspectives in Psychiatric Care, 41, 1-13. doi:10.1111/j.0031-5990.2005.00006.x

[32] Meehan, D.B. (2012) Phenomenal space and the unity of conscious experience.

http://psyche.cs.monash.edu.au/v9/psyche-9-12-meehan.h $\underline{\mathrm{tml}}$

[33] Mishler, E.G. (1986) Research interviewing: Context and narrative. Harvard University Press, London.

[34] Granheim, U.H. and Lundman, B. (2004) Qualitative content analysis in nursing research: Concepts, procedures and measures to achieve trustworthiness. Nurse Education Today, 24, 105-112. doi:10.1016/j.nedt.2003.10.001

[35] Hem, M.H. and Heggen, K. (2003) Being professional and being human: One nurse's relationship with a psychiatric patient. Journal of Advanced Nursing, 43, 101-108. doi:10.1046/j.1365-2648.2003.02677.x

[36] Folstein, M.F. (1983) The mini-mental state examination. In: Crook, T., Ferris, S. and Bartus, R., Eds., Assessment in Geriatric Psychopharmacology, Mark Powley, New Canaan, 50-51.

[37] Van Manen, M. (2007) Researching lived experience. Human science for an action sensitive pedagogy. The Althouse Press, Ontario.

[38] Zingmark, K., Norberg, A. and Sandman P.O. (1995) The experience of being at home throughout the life span. Investigation of persons aged from 2 - 102. International Journal of Aging and Human Development, 41, 47-62. doi:10.2190/N08L-42J5-31D2-JUQA

[39] Romme, M., Escher, S., Dillon, J., Morris, M. and Corstens, D. (2009) Living with voices: 50 stories of recovery. Person Centered Counseling Services Books, Herefordshire.

[40] Beavan, V. and Read, J. (2010) Hearing voices and listening to what they say: The importance of voice content in understanding and working with distressing voices. Journal of Nervous and Mental Disease, 198, 201-205. doi:10.1097/NMD.0b013e3181d14612

[41] Barrett, T.R. and Caylor, M.R. (1998) Verbal hallucinations in normals, V: Perceived reality characteristics. Personality and Individual Differences, 25, 209-221. doi:10.1016/S0191-8869(98)00014-2

[42] Freeman, D., Garety, P.A., Fowler, D., Kuipers, E., Bebbington, P.E. and Dunn, G. (2004) Why do people with delusions fail to choose more realistic explanations for their experiences? An empirical investigation. Journal of Consulting and Clinical Psychology, 72, 671-680. 


\section{doi:10.1037/0022-006X.72.4.671}

[43] Martin, P.J. (2000) Hearing voices and listening to those that hear them. Journal of Psychiatric and Mental Health Nursing, 7, 135-141. doi:10.1046/j.1365-2850.2000.00276.x

[44] Smith, M.J., Thirthalli, J., Abdallah, A.B., Murray, R.M. and Cottler, L.B. (2009) Prevalence of psychotic symptoms in substance users: A comparison across substances. Comprehensive Psychiatry, 50, 245-250. doi:10.1016/j.comppsych.2008.07.009

[45] Kiekkas, P., Theodorakopoulou, G., Spyratos, F. and Baltopoulos, G.I. (2010) Psychological distress and delusional memories after critical care: A literature review. International Nursing Review, 57, 288-296. doi:10.1111/j.1466-7657.2010.00809.x

[46] Friedman, J.H. (2010) Parkinson's disease psychosis 2010: A review article. Parkinsonism and Related Disorders, 16, 553-560. doi:10.1016/j.parkreldis.2010.05.004

[47] Parse, R.R. (1998) The human becoming school of thought. Sage, Thousand Oaks.

[48] Coleman, R. (1999) Recovery: An alien concept. Handsell Publishing, Gloucester.

[49] Millham, A. and Easton, S. (1998) Prevalence of auditory hallucinations in nurses in mental health. Journal of Psychiatric Mental Health Nursing, 5, 95-99. doi:10.1046/j.1365-2850.1998.00098.x

[50] Adam, R., Tilley, S. and Pollock, L. (2003) Person first: What people with enduring mental disorders value about community psychiatric nursing and CPN services. Jour- nal of Psychiatric Mental Health Nursing, 10, 203-212. doi:10.1046/j.1365-2850.2003.00560.x

[51] Carlsson, M.E. and Nilsson, I.M. (2007) Bereaved spouses' adjustment after the patients' death in palliative care. Palliative and Supportive Care, 5, 397-404. doi:10.1017/S1478951507000594

[52] Baethge, C. (2002) Grief hallucinations: True or pseudo? Serious or not? Psychopathology, 35, 296-302. doi:10.1159/000067067

[53] Kersting, A. (2004) The psychodynamics of grief hallucinations-A psychopathological phenomenon of normal and pathological grief. Psychopathology, 37, 50-51. doi:10.1159/000077020

[54] Simon, N.M., Wall, M.M., Keshaviah, A., Dryman, M.T., LeBlanc, N.J. and Shear, M.K. (2011) Informing the symptom profile of complicated grief. Depression and Anxiety, 28, 118-126. doi:10.1002/da.20775

[55] Thomas, P. and Bracken, P. (2004) Hearing voices: A phenomenological-hermeneutic approach. Cognitive Neuropsychiatry, 9, 13-23. doi:10.1080/13546800344000138

[56] Guba, E.G. (1981) Criteria for assessing the trustworthiness of naturalistic inquiries. Educational Technology Research and Development, 29, 75-91.

[57] Diederen, K.M.J., van Lutterveid, R. and Sommer, E.C. (2012) Neuorimaging of voice hearing in non-psychotic individuals: A min-review. Frontiers of Neuroscience, $\mathbf{6}$, 111. doi:10.3389/fnhum.2012.00111 